\title{
"SIN NOSOTRAS NO HAY OLLAS": ANÁLISIS DESDE LOS MERENDEROS, COMEDORES Y SUS TRABAJADORXS EN RELACIÓN A LA ECONOMÍA POPULAR.
}

\author{
Juliana Aloi ${ }^{1}$
}

\section{Introducción}

Antes de comenzar a desarrollar el análisis de este trabajo, me gustaría introducir el lugar a partir de donde me encuentro escribiendo para poder dar cuenta así de mi relación con la temática y la construcción del interés sobre esta problemática.

Desde el año 2016 formo parte del equipo de Economía Popular, Trabajo y Territorio que funciona en una sede de la Facultad de Filosofía y Letras (FFyL) de la Universidad de Buenos Aires (UBA): el Centro de Innovación para el Desarrollo y la Acción Comunitaria (CIDAC) ${ }^{2}$. Este se encuentra ubicado en el barrio porteño de Barracas, al sur de la ciudad. Como grupo, venimos trabajando en diferentes dispositivos y proyectos. Uno de ellos consiste en un relevamiento permanente de distintos espacios productivos de la economía popular en la comuna 4. En ese marco, surgieron como espacios relevados algunos merenderos y comedores. Si bien en una primera instancia no fue una pregunta en la cual hayamos profundizado, hoy individualmente retomo ciertas cuestiones como ¿por qué surgen los merenderos como espacios productivos? ¿qué producen? Otro claro interés, también relacionado con mi trayectoria en el equipo, es la motivación por buscar una perspectiva de género en la producción de conocimiento relacionado al campo abordado académicamente de la Economía Popular.

A partir de la economía de los cuidados y los aportes de la economía feminista, la gran cantidad de mujeres que participan en organizaciones sociales y

\footnotetext{
${ }^{1}$ Universidad de Buenos Aires, Argentina. Email: aloi.juliana@gmail.com ORCID id: https://orcid.org/0000-0003-4171-0976

2 Instituto de Ciencias Antropológicas. Proyecto UBACyT "Renovadas condiciones de explotación, disputas politicas y reconfiguraciones actuales en el mundo del trabajo".
} 
desempeñan tareas de cuidados, encontré en los merenderos y comedores un dispositivo pertinente de análisis. Es en parte por esto que surge mi interés acerca de indagar sobre estos espacios. A su vez, mis producciones teóricas se enmarcan dentro de un proyecto UBACYT a cargo de la Dra. Ivanna Petz titulado "Renovadas condiciones de explotación, disputas políticas y reconfiguraciones actuales en el mundo del trabajo", es en consonancia con este proyecto y a la trayectoria mencionada que desarrollo mi marco epistemológico, teórico y político.

Siguiendo lo introducido anteriormente, en esta oportunidad me interesa relatar en primer lugar sobre mi acercamiento a la temática. A su vez, empezar a realizar una descripción de los merenderos, los comedores y su relación con la Economía Popular. Para esto limitaré en primera instancia mi campo de acción e investigación a aquellos espacios que estén relacionados con la Confederación de Trabajadores de la Economía Popular (CTEP) y que se encuentren en la Ciudad Autónoma de Buenos Aires (CABA).

Los primeros interrogantes que me llevaron a acercarme al territorio en cuestión estuvieron relacionados con la situación actual de los merenderos y comedores. Con esto me refiero al período de gobierno nacional de Mauricio Macri (2015-2019) donde la situación económica y social se vió muy deteriorada.

Realicé algunos acercamientos a territorio, visitando merenderos y comedores de distintas comunas. También cuento con insumos producto de diversas conversaciones y entrevistas a personas afines a la temática. Por lo tanto utilizo como material de análisis, registros de campo, registros de charlas/conferencias, entrevistas y bibliografía de distintos autores.

\section{La Economía Popular y la CTEP.}

Para comenzar el desarrollo de este trabajo quisiera discutir algunos puntos sobre aquellos sujetos que conforman el campo de lo que llamamos economía popular (EP). En primer lugar, mencionar que la utilización de la categoría economía popular es en parte una categoría política reivindicativa. Este término no 
surge específicamente de la Academia, sino que es utilizado por los propios sujetos, según Grabois y Pérsico ${ }^{3}$ (2014) estos son:

\begin{abstract}
los compañeros que tuvieron que salir a inventarse el trabajo, revolver la basura en la noche fría para juntar plástico, papel y cartón, pasar noches en vela para recuperar la empresa quebrada, vender baratijas en trenes y colectivos, aprender a producir artesanías para subsistir, tirar la manta en la calle frente a la mirada adusta de la policía, bancar la parada en la feria, salir con el carro a caballo a fletear, subirse a la moto arriesgando la vida para llevar mensajes y encomiendas, cultivar la tierra frente a la amenaza constante del agronegocio, sostener un emprendimiento familiar ante la competencia de los capitalistas, pintar una escuela o barrer las calles por un mísero subsidio, cuidar chicos en el barrio, cocinar en los comedores, trabajar en espacios comunitarios... todos estos compañeros fueron creando, desde esos basurales sociales, ejemplos de trabajo, organización, lucha y dignidad. Lo que nosotros llamamos "Economía Popular" (Grabois y Pérsico, 2014: 2-3).
\end{abstract}

Creemos que la categoría de trabajo informal no logra incluir la totalidad de formas de empleo que ocurren al interior de la EP. Así como tampoco es una certeza, sino más bien un debate latente sobre si la categoría EP logra denominar o se ajusta a la realidad del sujeto en cuestión. Es posible decir que los trabajadores de la EP son informales, pero la dicotomía formal vs informal, deja por fuera toda la capacidad operatoria de la cual buscamos dar cuenta con nuestras producciones. Este razonamiento se asemeja a cuando Melliasoux (1977) realiza en su obra Mujeres, graneros y capitales una crítica a las visiones negativas que buscaban clasificar a las comunidades domésticas por lo que no son: "(...) se reduce a una demostración de una perspectiva limitada, a saber: que esas sociedades precapitalistas sólo serían diferentes del capitalismo porque son su revés" (Melliasoux, 1977: 16). Es decir que la EP no puede reducirse únicamente a aquello que no es trabajo formal. Es una porción fundamental del mundo del trabajo.

En este punto, me interesa retomar a ciertos autores del campo de la Antropología Económica y de otras disciplinas sociales como aporte analítico para entender a la Economía Popular. Uno de ellos es el antropólogo Hugo Trinchero (1995), quien va a proponer extender la categoría de "economía doméstica" utilizada para caracterizar a la forma de organización de los modos de producción de familias campesinas, a las dinámicas económicas populares en contextos urbanos. Pensando en las formas de organización de las unidades domésticas campesinas es que se

3 Juan Grabois pertenece al Movimiento de Trabajadores Excluídos (MTE), el cual forma parte de la CTEP. Emilio Pérsico es uno de los fundadores del Movimiento Evita, el cual también pertenece a la CTEP. Desde sus organizaciones, ambos son dirigentes reconocidos de la CTEP. 
traspola esta categoría y se extiende a otros contextos, como los urbanos. La categoría EP engloba un campo heterogéneo por diversas situaciones y niveles de relacionamiento (funcionalidad, contradicción, autonomía, etc.) respecto del proceso de reproducción simple y ampliada del capital. Es así que entendemos a todas aquellas formas denominadas procesos económicos populares como dinámicas económicas que se conforman como parte estructural del proceso de acumulación capitalista y no por fuera de él.

Parte de esta heterogeneidad se puede ver en la estructura de organización de la CTEP, por ejemplo. Según las descripciones propias de la Confederación, "la CTEP es una herramienta de lucha reivindicativa para la restitución de los derechos laborales y sociales que nos arrebató el neoliberalismo y que aún no hemos recuperado" ${ }^{4}$. Para entender mejor a la organización de la CTEP, retomo a Larsen (2016):

De la CTEP participan diferentes fuerzas políticas y sociales, nucleadas en diferentes Federaciones, según rama de actividad (...) Además de la división en federaciones, existe un espacio en la estructura organizativa denominada Junta Promotora, que nuclea a todas las organizaciones sociales integrantes. Algunas de estas organizaciones son: Movimiento Evita, MTE, Marea Popular, Quebracho-CTD Aníbal Verón, MOCASEVíaCampesina, Movimiento Nacional Campesino Indígena, Movimiento Popular La Dignidad, Organización Política y Social Los Pibes, Frente Popular Darío SantillánMTD Aníbal Verón,entre las más referenciadas (Larsen, 2016: 2).

Esta Confederación de trabajadores surge en el año 2011. En Argentina existe una tradición sindical con muchos años de historia, dentro de las estructuras sindicales clásicas podemos distinguir a la Central General de Trabajadores (CGT) o la Central de Trabajadores Argentinos (CTA), al interior de estas se agrupan uniones obreras de diversos tipos de trabajos (construcción, ladrilleros, jaboneros, comercio, etcétera). Surge aquí una posible diferenciación con la CTEP, quienes se encuentran nucleadas en su interior son -como mencioné anteriormente- diferentes fuerzas políticas y sociales. El extenso grupo de trabajadores que podemos ubicar en la Economía Popular es un sujeto heteróclito. Es a este sujeto a quien busca organizar la CTEP, por eso es atinado pensar y reflexionar en las particularidades y diferencias con otras centrales sindicales como las mencionadas anteriormente. La división en

4 Nosotros, http://ctepargentina.org/nosotros/ 
ramas de actividad es útil para establecer un cierto orden en la diversidad de trabajos que podemos encontrar al interior de esta confederación. Algunas de estas ramas son Infraestructura; Vía Pública; Recicladores (cartoneros); Textil y Socio-comunitaria. En esta última rama es donde se encuentran los espacios que tomo como referentes empíricos para el actual análisis: los merenderos y los comedores.

Cada merendero y/o comedor, será interpretado como una unidad productiva (UP). Tomo esta categoría de la propia organización de la CTEP, donde se entiende a las UP como "el conjunto de los medios de producción (maquinarias, infraestructura, espacio, materias primas) que sumado a nuestra fuerza de trabajo permiten producir un bien o servicio" (Grabois y Pérsico, 2014). Como parte de la diversidad de unidades productivas podemos agrupar a cooperativas, fábricas recuperadas, emprendimientos familiares, y diversas formas de agrupación que responden a la heterogeneidad que compone a la EP. Estas unidades productivas se diferencian de lo que podemos entender como una empresa, una pyme o una fábrica. Desde un plano ideológico y político, pero fundamentalmente desde un plano material basado en las desigualdades que podemos encontrar entre estos. Las unidades productivas en mayor o menor medida, no cuentan con un gran capital inicial (como si se requiere para una empresa), grandes maquinarias o infraestructura. Así como tampoco tienen como objetivo primordial la búsqueda de la ganancia, sino que su fin son poder crear medios e ingresos para subsistir: la creación de las propias fuentes laborales. Podemos pensar entonces que estas unidades productivas son un ejemplo concreto de la organización de estos trabajadores de la EP que se agrupan para poder generar condiciones lo más dignas posibles para trabajar. Si pensamos en los comedores y merenderos, el trabajo que allí se lleva adelante a su vez está relacionado con un propósito social y comunitario: cuidar y alimentar a aquellos que lo necesiten en los distintos barrios de la ciudad. No solo son una forma de organización para el trabajo, sino que también son un medio para garantizar la subsistencia en este caso no exclusivamente la propia de los trabajadores y sus núcleos familiares, sino que también la del conjunto de la sociedad.

Es así que podemos empezar a ver en este sentido, la capacidad de agencia de quienes componen la EP. Buscamos diferenciarnos aquí de una mirada que minimice a estos sectores o piense que las relaciones entre estos y el Estado -por ejemplo- solo pueden venir de la mano de políticas de asistencialismo. Esta agencia es posible 
demostrarla a partir de distintos dispositivos, como la capacidad de producir estrategias de autoempleo o las distintas formas de organización y asociación (Petz; 2013 y 2016 y 2017, Petz y Larsen; 2015). Es de estas formas que buscaré dar cuenta centrándome en la rama Socio-comunitaria de la CTEP, en este trabajo puntualmente en los merenderos y comedores.

Por lo tanto, es posible decir que dentro de la EP se encuentran aquellas personas que no poseen relaciones laborales formales, quienes crean sus propias fuentes de trabajo y éstas en consonancia con distintas estrategias de organización. Siguiendo esta definición que fui construyendo en este apartado, también cabe destacar que no todas las personas que realizan tareas laborales que coinciden con las características aquí propuestas, se encuentran organizadas o se reconocen como trabajadores de la Economía Popular. Pero sí podemos decir que es a este sujeto al que busca organizar la CTEP. Es importante destacar también que estas diversas estrategias de trabajo no están por fuera de los patrones de acumulación actuales, sino todo lo contrario: son parte constitutiva de estos.

\section{Merenderos y comedores en la Ciudad Autónoma de Buenos Aires}

En esta oportunidad, como referente empírico de mi análisis elijo a los merenderos y comedores de la Ciudad Autónoma de Buenos Aires. Existen múltiples merenderos y comedores que forman parte de organizaciones sociales y políticas más amplias, es decir que se encuentran dentro de un entramado de relaciones y no son unidades "sueltas" e independientes. Una de estas "organizaciones sociales más amplias" es la CTEP.

La Ciudad de Buenos Aires se encuentra dividida en comunas a partir de la Ley de Comunas (Ley $\mathrm{N}^{\circ}$ 1777) que divide a la Ciudad de Buenos Aires en quince comunas, estas a su vez están integradas por barrios. Menciono esto ya que es importante para posicionarse espacialmente en los distintos merenderos y comedores que se pueden encontrar a lo largo de la Ciudad. 


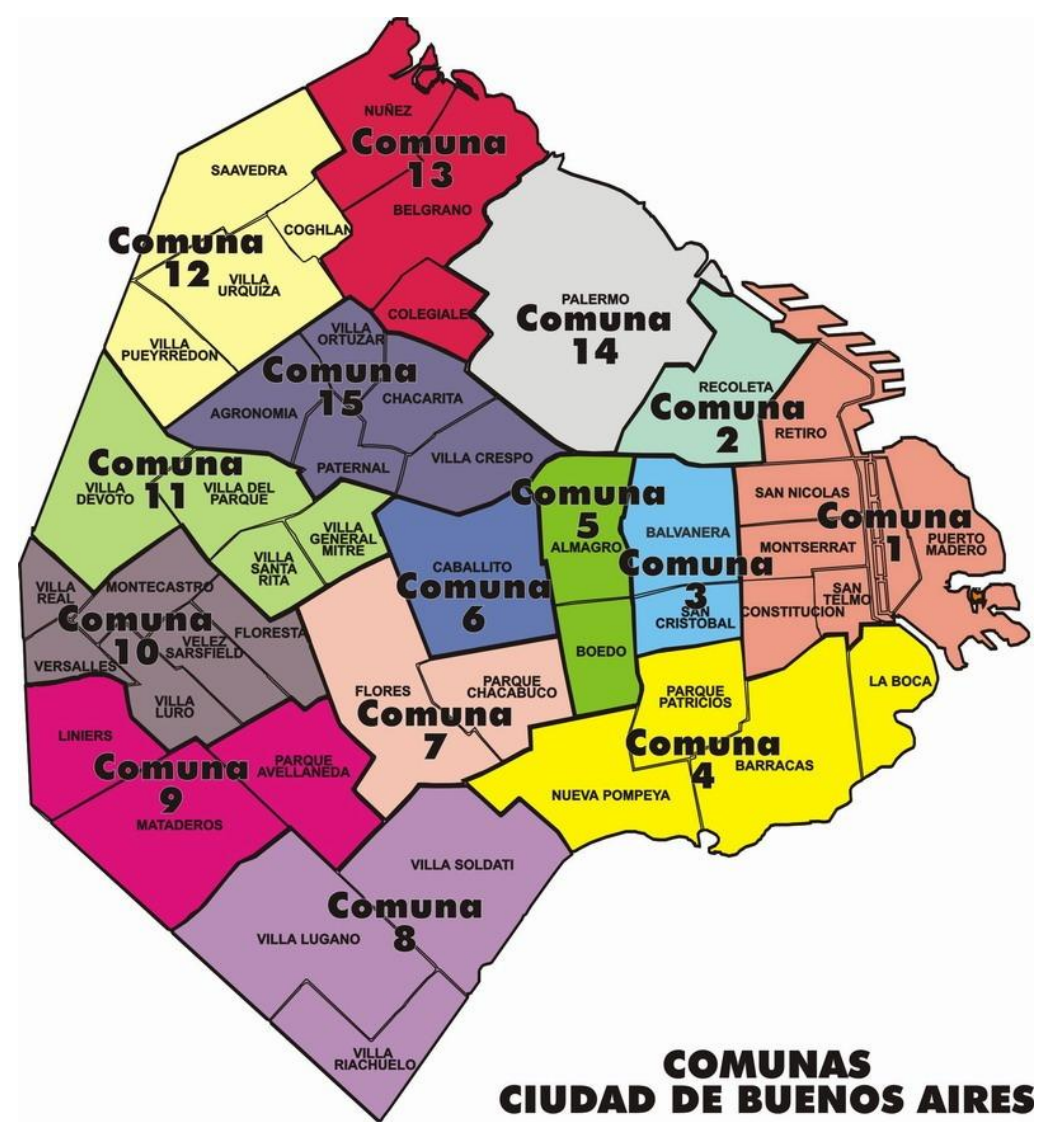

Mapa de la organización en comunas de la CABA, con sus respectivos barrios en cada una.

Podemos encontrar espacios de este tipo vinculados a instituciones de diversos sectores, como sectores religiosos; fundaciones y organizaciones no gubernamentales; movimientos sociales; y partidos políticos. Dentro de este vasto entramado es que selecciono a aquellos espacios con vinculación a la CTEP. La división en comunas, posiciona a cada una como una unidad de gestión política y administrativa con competencia territorial. Esta división es tomada a su vez por algunas organizaciones para su propia organización territorial, con respecto a su militancia y sus dispositivos de acción y participación. Siguiendo la organización de la CTEP, en cada comuna de la ciudad de Buenos Aires podemos encontrar varias unidades productivas. Es decir que es frecuente relacionar las actividades de un merendero o un comedor en el interior de la comuna en la que se encuentre, vinculandose con distintos espacios que puedan existir en ese territorio. Tanto del interior de la organización, como con otros agentes, por ejemplos clubes del barrio, escuelas o centros de salud comunitarios. 
Aquellos que llamamos merenderos suelen funcionar principalmente en el horario de la tarde, es decir entre las 16 y las 18 horas, por ejemplo. En cambio los comedores suelen funcionar en el horario de la cena, es decir un poco más tarde. Estos horarios son pertinentes en cuanto a la actividad específica de dar la merienda o la cena, respectivamente. Esta forma de funcionamiento suele aplicarse en términos generales en todos los merenderos y comedores de la ciudad. Pero en cada uno podemos encontrar distintas particularidades, es por esto que el aporte del trabajo de campo nos da un caudal de información sobre a la cotidianidad de cada uno. Es posible sumar a la descripción que, en estos espacios suelen funcionar otras actividades como apoyo escolar; talleres de arte o culturales; actividades relacionadas a la militancia como temas de género, formación sindical/gremial; entre muchas otras.

Para esta oportunidad tomaré el trabajo de campo realizado en dos merenderos y entrevistas a distintos referentes. Por un lado, uno ubicado en el barrio de Barracas, específicamente en la villa 21-24. Esto es al sur de la Ciudad dentro de la comuna 4, en uno de los barrios más abandonados por el gobierno de la ciudad. Por otro lado, visité un merendero dentro de la comuna 1 en el barrio de Constitución. Este barrio se caracteriza por ser un lugar de mucho tránsito de personas, ya que desde allí salen diversos medios de transporte que conectan la capital federal con el conurbano bonaerense.

A partir de una entrevista realizada al responsable de la rama Sociocomunitaria del Movimiento Evita ${ }^{5}$-CTEP de CABA pude obtener información en cuanto a la organización y composición de esta rama, en la cual se incluyen a los merenderos y los comedores. En la Ciudad Autónoma de Buenos Aires se estima que existe un número de quinientos espacios comunitarios ${ }^{6}$. Cabe aclarar que no existen datos oficiales y sistematizados por parte de organismos del Estado, sino que estas cifras han sido calculadas por diversas organizaciones sociales. Este bloque de trabajadorxs que conforman la rama está compuesto no solo por la CTEP sino que integra a una multiplicidad de organizaciones, como por ejemplo Barrios de Pie o la

5 Esta es una de las fuerzas políticas y sociales más grande dentro de la CTEP.

6 Esta categoría incluye a los merenderos, comedores, centros culturales y comunitarios. La retomo como categoría nativa, utilizada por los propios sujetos que componen estos espacios y la tomo como parte de mis explicaciones. 
Corriente Clasista y Combativa. La CTEP-Evita contiene en su interior una cantidad aproximada de 100 merenderos y comedores que se despliegan por todas las comunas de $\mathrm{CABA}$, desde la comuna 15 como en el barrio Chacarita hasta la comuna 1 como en el barrio de Constitución.

Luego de esta caracterización, podemos decir que al pensar en los merenderos y comedores, es posible ubicarlos como referentes empíricos de la EP. Al preguntarme ¿qué se produce allí? la primera respuesta que surge es que en verdad lo que allí ocurre es indudablemente trabajo reproductivo. En estos espacios suele haber una gran cantidad de mujeres trabajadoras que se ocupan de diversas tareas, principalmente de cocinar alimentos. También mantienen el orden y la limpieza de los lugares y cuidan constantemente de la seguridad de los niños. Al hablar de la reproducción de la fuerza de trabajo, se incluye a aquellas tareas que implican un trabajo de reproducción: cocinar, limpiar, procrear, cuidar (Federicci, 2018). Asimismo, lo que ocurre en los merenderos y comedores no se queda únicamente en el hecho de atender la emergencia del hambre y las tareas de cuidado. En estos dispositivos es posible dar cuenta de múltiples actividades y problemáticas que se atienden constantemente. En un principio al pensar la temática, mis preguntas estaban orientadas principalmente a cuestiones operativas del funcionamiento de los comedores y merenderos. Concentrándome específicamente en la tarea que estos llevan adelante con respecto a brindar una solución inmediata al hambre cotidiana de una gran cantidad de niños y niñas. Al acercarme al territorio, y hablar con las personas que sostienen estos espacios, pude dar cuenta que son unidades en donde transcurren múltiples actividades. Hacer un análisis sobre merenderos y comedores pensando únicamente en la actividad alimentaria, deja muchas cosas sin cubrir. Pude observar que en estos espacios ocurre una heterogeneidad de situaciones. Talleres de distintos oficios; actividades y talleres culturales; espacios de debate entre mujeres; etcétera.

En uno de los registros que realicé acudí a un merendero ubicado en la Villa 21-24 en el barrio de Barracas donde pude asistir a un encuentro de un grupo de mujeres del barrio que se juntan allí semanalmente para compartir sobre diversos temas de su interés, a partir de talleres o temáticas de discusión. En este mismo espacio, la responsable del merendero me contaba su interés en poder terminar de construir un baño en el inmueble en relación a la intención de poder realizar allí más 
actividades sin que eso sea un limitante para obligar a las personas a retirarse. Menciono esto a modo de ejemplo concreto, como símbolo de la meta de sus trabajadorxs de que estos sitios se constituyan como espacios de contención. Hay una intención visible por generar propuestas constantemente que van más allá del hecho de proporcionar una comida. En relación a esto, el responsable de la Rama Socio Comunitaria de la CTEP-Movimiento Evita, me decía que:

(...) su tarea, que no solo abarca el aspecto alimentario, sino que implica el atajar y el dar respuesta al conjunto de las problemáticas comunitarias que se expresan porque en los comedores, los merenderos, los compañeros, las compañeras, abordan las problemáticas educativas, sanitarias, los problemas vinculados a la violencia de género (extracto del registro realizado el 18/06/19, entrevista al responsable de la Rama Socio Comunitaria CTEP-Movimiento Evita).

Es decir que, la actividad concreta con la que son creados estos espacios es para atender la necesidad alimentaria de un amplio sector de la población que se encuentra en una estado de alta vulnerabilidad social. Pero, al mismo tiempo se posicionan como un lugar de referencia territorial en los distintos barrios donde se encuentran. Generando vínculos constantemente con otros actores y gestionando distintas estrategias de contención para abordar las demandas.

Siguiendo las reflexiones que Timpanaro y Spinosa (2018) realizan a partir de su trabajo en distintos espacios comunitarios donde se desarrollan tareas de cuidado en la zona noroeste del Gran Buenos Aires, es posible entender a los merenderos como "formas comunitarias y populares de trabajo, en la que territorio y trabajo se unen para dar lugar a distintas actividades, con valor económico y también social" (Timpanaro y Spinosa, 2018). Esto es visible a partir de las distintas situaciones que fui dando cuenta, los merenderos y comedores son un espacio importante en las realidades cotidianas de los sujetos que atienden y se posicionan como un espacio de referencia, contención e intervención en los distintos barrios de la Ciudad. Destaco el concepto de intervención ya que una de las cosas que pude observar es que gran parte de lxs trabajadorxs de estos espacios son a su vez los protagonistas de distintas luchas. En las cocinas de estos lugares no solo se cocinan alimentos sino que también se cocinan reivindicaciones sociales constantemente.

Pensar entonces en las distintas actividades y problemáticas que interpelan diariamente a las personas que acuden a los merenderos y comedores, implica necesariamente pensar en los trabajadores que sostienen estos espacios y las 
organizaciones que los contienen. Y a su vez en la situación concreta y real que atraviesan, surge aquí la pregunta de ¿cómo impacta la situación actual del país en estos espacios comunitarios? Al hacer referencia a la situación actual del país, me refiero a un deterioro de las condiciones de vida y un aumento progresivo de las tasas de desempleo. Siguiendo los datos difundidos por el Instituto Nacional de Censos y Estadísticas (INDEC) en el primer trimestre del año 2018 la tasa de desocupación (a nivel nacional) se encontraba en 9,1\%, aumentando hacia el primer trimestre del corriente 2019 hasta un 10,1\%. Estos datos cuantitativos, se reflejan constantemente en el análisis cualitativo que fui realizando en mis registros. En todos surgió la cuestión de que en los últimos años se multiplicó la cantidad de personas asistentes a estos espacios comunitarios, en gran medida pensados principalmente para niños. La realidad actual es que acuden familias enteras, adultos, niños y ancianos. Una de las responsables daba cuenta de como distintas personas incluso modifican notablemente su recorrido para volver a sus casas, con la intención de poder pasar por distintos puntos de la ciudad donde conseguir un plato de comida. Esto ocurre en la ciudad más rica del país, donde se registra actualmente un número de aproximadamente 600 mil pobres, con una línea de pobreza registrada en mayo de $21642,10^{7}$ que escala progresivamente. Un ejemplo puede verse reflejado en este extracto:

\begin{abstract}
hay una serie de problemáticas que la economía popular nace buscando resolver y como parte de su organización. Esas problemáticas no aparecieron con este gobierno, no nacen con este gobierno, PERO SI VINIERON A AGRAVARSE DE MANERA SUSTANTIVA EN EL CONJUNTO DE LOS BARRIOS DEL PAÍS. ::Eh:: ( ) está claro que el nivel de impacto directo en lo que es el desempleo ::eh:: ( ) Donde en los espacios que empezaron a venir los chicos y las chicas después del colegio, como una actividad más y hasta un poco como un simple complemento a lo que ellos ::ya::... ( ) no necesitaban de manera tan urgente AHORA NO SOLO VIENEN ESOS CHICOS Y ESAS CHICAS, SINO QUE VIENEN LOS PAPÁS, LAS MAMÁS, LOS ABUELOS, LAS ABUELAS, VIENE EL CONJUNTO DE LA FAMILIA. (extracto del registro realizado el 18/06/19, entrevista al responsable de la Rama Socio Comunitaria CTEPMovimiento Evita).
\end{abstract}

El gobierno nacional y el gobierno de la ciudad, destinan una cantidad de recursos en alimentos a algunos espacios comunitarios, producto en parte de las

7 Para un hogar tipo compuesto por una pareja de una mujer y un varón de 25 años de edad, ambos económicamente activos y no propietarios de la vivienda. Según la Dirección General de Estadística y Censos (Ministerio de Economía y Finanzas GCBA). 
jornadas de movilización ocurridas en el año 2018 bajo el nombre de Merendazo. Igualmente, estos recursos son insuficientes y se precisa constantemente de donaciones externas o del propio aportes de sus trabajadores o las organizaciones que contienen estos espacios.

Esto guía el análisis entonces a preguntarme nuevamente sobre las personas concretas que sostienen y llevan adelante estos espacios. Para profundizar en los trabajadores que componen los merenderos y comedores, es necesario hacer referencia a la notable cantidad de mujeres que desempeñan las tareas de cuidado que allí se llevan adelante. Son ellas quienes corporalizan (Rita Segato, 2013; Gago y Cavallero, 2019) la sostenibilidad de la vida (Rodriguez Enrique, 2015), la crisis y la demanda. A partir de la lectura de estas autoras, es posible decir que al pensar en las situaciones de crisis, las mujeres sufren en una mayor medida el impacto. De todas las personas que sufren los avances de políticas neoliberales, las mujeres son quienes más reciben el golpe. En consonancia con lo trabajado en esta oportunidad, los merenderos y comedores se convierten en un espacio fundamental para la supervivencia de las personas. En estos últimos cuatro años se multiplicó la demanda y los frentes a atender. Las altas tasas de desempleo y el incremento de la pobreza afecta en gran medida al sector. Una de las mujeres con las cuales tuve la oportunidad de conversar me comentaba que "económicamente nos están violentando (...) el trabajo es lo que falta" (extracto del registro realizado el 18/05/19, Merendero "Mi Cielo", Villa 21-24, Barracas). Las mujeres nuevamente salen a ocupar el rol de sostener la vida a partir de las tareas de cuidado, de hacerse cargo de la ausencia estatal: "sin nosotras no habría ollas (...) somos nosotras las que hacemos lo que no hace el Estado" (extracto del registro realizado el 2/05/19, Merendero "Corazón Valiente", Constitución). En este mismo lugar su referenta ${ }^{8}$ me comentaba que el $80 \%$ de las personas que desempeñan tareas son mujeres y que la mayoría de los compañeros varones son personas jóvenes que "se adaptan, entienden" (fragmento del registro del campo del día jueves 2 de Mayo) haciendo referencia a que constantemente en ese espacio se habla y reflexiona sobre la violencia machista. Rodriguez Enrique (2015) propone analizar la desigualdad de género a partir de un análisis sobre las tareas de cuidados y su organización, como determinante de la

8 Utilizo la palabra referenta en parte como categoría utilizada por los propios sujetos de la organización, y en parte como reivindicación política de las mujeres en espacios de liderazgo. 
desigualdad. Introduce el concepto de feminización de la pobreza, donde las mujeres desarrollan tareas que implican la reproducción de la fuerza de trabajo. Es posible observar esto tanto al interior de los hogares y familias como en los comedores y merenderos que me propongo investigar. El cuidado está presente aquí, pero desde un lugar de cuidado comunitario. Esta autora hace referencia a la existencia de una red de cuidados compuesta por distintos actores, como el Estado, las organizaciones, instituciones y cuidadores.

Gago y Cavallero (2019) sostienen que existe una politización de la crisis de reproducción donde hay un gran avance de la inseguridad alimentaria y una clara jerarquía entre lo productivo y lo reproductivo. Retomo de estas autoras el concepto de terror financiero que implica una estructura de obediencia sobre el día a día que obliga a asumir de manera individual y privada -y agregaría, comunitaria- los costes del ajuste. Es notable como muchas organizaciones sociales asumen estos costes permanentemente. En este caso de análisis encarnado en los espacios comunitarios, sostenidos mayoritariamente por mujeres trabajadoras. A partir de todo lo aquí recapitulado, es posible empezar a diagramar el lugar que salen a ocupar estos espacios comunitarios día a día frente a la desidia estatal. Donde a partir de la posición y la trayectoria construida con las poblaciones de los distintos barrios de la Ciudad, se constituyen como un lugar de referencia donde acudir frente a problemáticas tales como violencia de género, consumo problemático, etcétera. Problemáticas que se encuentran en un momento de auge frente a momentos históricos nacionales de situaciones económicas inestables.

\section{Algunas conclusiones y nuevas preguntas...}

A partir de los primeros acercamientos al campo que fui realizando, desde las primeras instancias en la cual empecé a sistematizar y esbozar algunas conclusiones, puedo decir que intenté lograr ver toda una totalidad en donde los merenderos y 
comedores sean interpretados como un referente empírico más de un entramado que incluye a muchos más dispositivos, problemáticas y demandas.

Para entender la realidad como un todo no es suficiente ver las relaciones, hechos y procesos, sino que es necesario a su vez tener en cuenta su creación, su estructura y su génesis (Kosik, 1967). Me parece adecuado destacar la importancia de no estudiar los fenómenos aislados. En un principio empecé a analizar a los merenderos y comedores con una visión quizás un poco cerrada a ese referente empírico. Con los diversos acercamientos al campo, fui dejando de lado ciertos presupuestos y también surgieron otras formas de interpretar a esos espacios, con estrecha relación a las demandas del sector de la EP, a sus conquistas a lo largo de las movilizaciones y luchas dadas, como la Ley de Emergencia Social aprobada en el año 2016, de donde surge el salario social complementario, el cual perciben muchos de los trabajadores de los merenderos y comedores. Esto a su vez inscripto en los debates y demandas históricas dados al interior de la CTEP como el reconocimiento de la condición de trabajadores de aquellos que no lo hacen de una manera formal. Y a su vez, la demanda por el reconocimiento de los distintos derechos conquistados a lo largo de la historia por los trabajadores en relación a condiciones dignas de empleo, jornadas laborales de una determinada cantidad de horas, paritarias, y lo que podemos denominar como salario indirecto. Es importante estudiar todos los elementos de la realidad, los merenderos y comedores, sus trabajadorxs, las organizaciones sociales que los contienen, las demandas y conquistas del sector, el rol del estado expresado por ejemplo en el Ministerio de Salud y Desarrollo Social de la Nación -por nombrar a uno de los organismos pertinentes-, los representantes y referentes políticos, etcétera. Reitero el énfasis por lo tanto en la importancia de analizar el problema en términos relacionales como propone Kosik.

Como parte de esta realidad concreta es que creo adecuado mencionar para futuros análisis la aprobación reciente de la Ley de Emergencia Alimentaria, aprobada durante el mes de Septiembre del año 2019. Esta fue una conquista de los movimientos populares, de los cuales la CTEP forma parte fervientemente. Como parte de los comunicados oficiales de la CTEP destacan que: 
la producción, distribución y al precio de los alimentos. (http://ctepargentina.org/seaprobo-unanimidad-la-emergencia-alimentaria/).

Esto habilita a seguir profundizando sobre la pregunta acerca de la producción, lo productivo y cómo esta temática esta presente en el sector de la EP. A su vez, nos vemos frente a un nuevo escenario político en donde otra fuerza de gobierno con un proyecto diametralmente distinto ha ganado las elecciones nacionales para los próximos cuatro años (2020-2024). Esto es bajo la fórmula peronista de Alberto Fernández ${ }^{9}$ y Cristina Fernández de Kirchner $^{10}$, la cual se caracterizó por lograr un espacio de unidad llamado "Frente de Todos" donde se incluye a distintos referentes de lo que podemos llamar el campo de lo nacional y popular. El presidente electo como una de sus principales propuestas de campaña presentó el programa "Argentina contra el hambre", donde se propone entre otras cosas:

compras a cargo del Estado a los productores de la economía social y popular, de la agricultura familiar y campesina, y a micro y pequeñas empresas; la sanción de una Ley de Góndolas, para garantizar variedad de marcas en los productos alimenticios; mercados locales de cercanía, almacenes campesinos, y ferias populares para el mercado interno; créditos a tasas bajas e incentivos a la economía social y la agricultura familiar; redes locales de comercialización (Qué es el plan "Argentina contra el hambre", https://www.redaccion.com.ar/que-es-el-plan-argentina-contra-el-hambre/).

Como así también: "mejorar la alimentación y la nutrición", "bajar el precio de los alimentos", "crear empleos", "implementar un sistema de alimentación sustentable y sostenible desde la producción hasta el consumo" (Cuáles son los principales puntos del programa "Argentina contra el Hambre",

\section{https://www.ambito.com/cuales-son-los-principales-puntos-del-programa-argentina-} contra-el-hambre-n5058601)

Creo fundamental hacer énfasis en la capacidad de agencia de la heterogeneidad de sujetos que componen a la EP en cuanto a dar respuestas políticas frente a una limitada presencia estatal. Entendiendo a lo político en términos abarcadores, contemplando factores económicos, culturales y sociales. Lo político como el reflejo de las distintas instancias del poder social. La lógica que rige lo político es de cooperación o antagonismo entre voluntades colectivas, donde se

9 Abogado y profesor, quien se desempeñó como Jefe de Gabinete durante del gobierno de Néstor Kichner.

10 Abogada, quien fue presidenta de Argentina durante dos mandatos, desde el 10 de diciembre de 2007 hasta el 9 de diciembre de 2015, obteniendo la reelección luego de su primer mandato. 
incorpora esquemas de alianzas y proyectos de acción (Argumedo, 2001). Bajo esta perspectiva es que la historia la interpretamos como un proceso de enfrentamientos o acuerdos de intereses donde se enfrentan fuerzas sociales y proyectos políticos. Es dentro de ese entramado donde se tiende a ampliar los espacios de la autocreación popular (Wilmer, 1975; Argumedo, 2001). Esta limitada ausencia estatal en cuanto a un escaso o nulo financiamiento a estas unidades productivas y a la falta de respuestas y soluciones, ocurre al mismo tiempo de una fuerte presencia estatal en los distintos barrios populares de la ciudad pero con otros dispositivos como por ejemplo el despliegue de fuerzas de seguridad. Es importante ver entonces la existencia de una compleja correlación de fuerzas, donde diferentes sujetos sociales buscan tener injerencia.

Es en esta correlación de fuerzas donde estos grupos de trabajadores actúan. A partir de la creación de nuevos espacios comunitarios, donde no solo se da una respuesta al hambre y a las diversas problemáticas, sino que se organizan al interior como trabajadores de la EP conformándose en distintas unidades productivas. Esto también es visible en la conformación de la Rama Sociocomunitaria y la lucha llevada adelante en el marco de la sanción de la Ley de Emergencia Social en el año 2016. Es decir que puede observarse esta capacidad de agencia del sector en las distintas medidas como la elaboración de proyectos de ley (emergencia social, alimentaria, de género, de adicciones); en las diversas campañas llevadas adelante; y en un permanente estado de movilización para exigir al Estado expresado en distintos organismos (ANSES, Ministerio de Desarrollo, etcétera) que legisle en consonancia con sus demandas. Retomo aquí el concepto de acontecimiento que propone Alcira Argumedo (2001), quien a su vez retoma a autores como Nun (1989), donde puede pensarse a estos como el ocurrir de determinados hechos o procesos que generan una nueva dinámica en el accionar político y social. Entendiendo a estos acontecimientos como determinados hechos detonantes que producen un escenario diferente en la vida social. Estos sucesos son sin dudas el resultado de un proceso histórico, pero con un fuerte accionar en el presente histórico.

Es entonces que los merenderos y comedores los tomo como un eje de análisis en relación constante y como parte constitutiva de la Economía Popular, con una importante presencia de la gremial organizadora que es la CTEP. Ni los merenderos ni los comedores fueron creados puntualmente por este sujeto, pero en 
los últimos años la CTEP y las organizaciones populares cobraron una presencia importante en la agenda política y estatal, reflejado constantemente en las calles. La intervención de la EP a partir de la CTEP y las distintas organizaciones sociales que la integran y/o acompañan, se torno un hecho fundamental frente a este escenario político particular.

Para ir finalizando, considero fundamental seguir reflexionando sobre el gran número de trabajadoras que sostienen estos espacios. Donde nuevamente se invisibilizan gran parte de sus tareas. Si bien es un hecho que gran parte percibe un salario social ${ }^{11}$ por las tareas que desempeñan, no hay una dimensión que tenga en cuenta el "valor económico del cuidado" (Alejandro Barrios, 2019). Estas trabajadoras se encuentran en cierto punto frente a una doble invisibilización, como trabajadoras de la economía popular quienes se encuentran en una lucha permanente por el reconocimiento de sus derechos y frente al hecho de ser mujeres haciéndose cargo de tareas de reproducción históricamente minimizadas. Pero al mismo tiempo, esta doble invisibilización también creo adecuado pensarla como una doble lucha que encara este grupo de mujeres trabajadoras. Mujeres que ponen el cuerpo, en varios sentidos. Con su fuerza de trabajo cocinando y atendiendo los merenderos y con su militancia que resiste constantemente los costes del ajuste.

Es así posible concluir que estos espacios son de vital importancia al interior de las distintas realidades territoriales. Podemos pensar que funcionan como un diagnóstico de las distintas problemáticas que ocurren en los territorios. Problemáticas de todo tipo, siendo la urgente a atender el hambre. Al estar inmersas en una organización existe un esquema complejo de relaciones, donde surge fuertemente la identidad de trabajadores de aquellas personas que sostienen estas unidades. Cabe preguntarse entonces qué relación hay entre lo productivo y estos espacios ¿queda sujeto únicamente a lo reproductivo? Esto último sin dudas está presente en las tareas diarias que encarar estos trabajadores y trabajadoras. A la vez me pregunto ¿cómo se construyó esta identidad como trabajadores y trabajadoras? ¿qué rol ocupó y ocupa en este caso la rama sociocomunitaria de la CTEP? y, ¿otros factores como el salario social complementario en relación a la ley de emergencia

11 El salario social complementario (SSC) es una de las medidas que forma parte de la Ley de Emergencia Social. Este es percibido por un gran número de personas que cumplen con determinados requisitos, y consta del $50 \%$ del salario mínimo vital y móvil. 
social? ¿Qué rol ocuparon las unidades sociocomunitarias en la Ley de emergencia Alimetaria? Y por último, ¿qué rol ocuparán estas organizaciones frente al nuevo escenario político y social?

\section{REFERENCIAS}

ARGUMEDO, Alcira. Los Silencios y las Voces de América Latina. Notas sobre el pensamiento nacional y popular. Buenos Aires: Ediciones del pensamiento nacional, Ediciones Colihue. 2001.

BARRIOS, Alejandro. La economía, el trabajo, y el cuidado. 2019. En BRANDARIZ, Carolina. No es amor. 2019. Buenos Aires: Editorial Indómita Luz.

CAVALLERO, Luci y GAGO, Verónica: Una lectura feminista de la Deuda: ¡vivas, libres y desendeudadas nos queremos! 1ra edición. Ciudad Autónoma de Buenos Aires: Fundación Rosa Luxemburgo, 2019.

FEDERICCI, Silvia: El patriarcado del salario. Críticas feministas al marxismo. 1ra edición. Ciudad Autónoma de Buenos Aires: Editorial Tinta Limón, 2018.

FEDERICCI, Silvia: Revolución en punto cero. 1ra edición. Ciudad Autónoma de Buenos Aires: Editorial Tinta Limón, 2018.

LARSEN, Matías. Los trabajadores de la CTEP y el Estado: algunas reflexiones desde la antropología económica. Jornadas de Investigación Santiago Wallace, 2016.

PERSICO E. y GRABOIS, J (2014) Organización y Economía Popular. Nuestra realidad. No 1, 2, 3 y 4, Buenos Aires, CTEP - Asociación Civil de los Trabajadores de la Economía Popular.

PETZ, I (2016) De poblaciones inempleables a trabajadores de la economía popular. Configuración de la trama de la economía popular en la Argentina actual. Informe presentado a Conicet, MINCyT.

PETZ, I. (2014) Acerca del marco interpretativo que orienta el área de economía popular del CIDAC. Ponencia presentada en el XI Congreso Argentino de Antropología Social. 23 al 26 de Julio. Facultad de Humanidades, Universidad Nacional de Rosario.

PETZ, I. (2013) La economía social y la reactualización del debate formalistassustantivistas". Ponencia presentada en las VII Jornadas de Investigación en 
Antropología Social. 22, 23 y 24 de Noviembre, Instituto de Cs Antropológicas, FFyLUBA.

RODRIGUEZ, Corina. Economía feminista y economía del cuidado. Aportes conceptuales para el estudio de la desigualdad. Revista Nueva Sociedad N 256, marzoabril de 2015, ISSN: (0251-3552, www.nuso.org)

TRINCHERO, Hugo (s.d.) Economía política de la exclusión. Para una crítica desde la experiencia de las empresas recuperadas por sus trabajadores. (ERT), Buenos Aires, 2007.

TIMPANARO, Berenice y SPINOSA, Lucas. 2018. Puentes entre la Economía Popular y la Economía Feminista. Experiencias de organización popular para la redistribución de las tareas de cuidado en el conurbano bonaerense. En BRANDARIZ, Carolina. No es amor. 2019. Buenos Aires: Editorial Indómita Luz.

\section{Fuentes}

\section{LEY DE EMERGENCIA SOCIAL Y ORGANIZACIONES DE LA ECONOMÍA POPULAR. Res. 1727/15MTEySS}

Encuesta EPH, Informes Técnicos. Vol. 3, nº 59 ISSN 2545-6636. Incidencia de la pobreza y la indigencia en 31 aglomerados urbanos. Segundo semestre de 2018 ISSN 2545-6660.

Líneas de indigencia (LI) y de pobreza (LP) para hogares tipo, en pesos. Ciudad de Buenos Aires. Enero de 2016/Mayo de 2019

Anuario Estadístico Ciudad de Buenos Aires, 2014. Dirección General de Estadísticas y Censos de la Ciudad de Buenos Aires.

Javier Sinay (7 de octubre de 2019). Qué es el plan "Argentina contra el hambre. RedAcción. Recuperada desde https://www.redaccion.com.ar/que-es-el-plan-argentinacontra-el-hambre/.

24 de noviembre de 2019. Cuáles son los principales puntos del programa "Argentina contra el Hambre". Ámbito Financiero. Recuperada desde https://www.ambito.com/cuales-son-los-principales-puntos-del-programa-argentinacontra-el-hambre-n5058601 